Ann. Biol. anim. Bioch. Biophys., I968, 8 (I), 8I-85.

NOTE ANNEXE

\title{
DÉTERMINATION DES CORPS CÉTONIQUES DANS LES LIQUIDES BIOLOGIQUES
}

\author{
R. PUECH \\ avec la collaboration technique de Nicole Picot et Marguerite Naville \\ Laboratoire de Recherches sur la Conservation et l'Efficacité des Aliments, \\ 16, rue Claude Bernard, Paris $5^{\mathbf{e}}$, \\ Centre national de Recherches zootechniques, \\ Institut national de la Recherche agronomique
}

\section{SOMMAIRE}

Des modifications sont apportées à la technique proposée par REID pour le dosage des substances cétoniques dans des liquides biologiques (sang, urine, contenu de rumen, ensilage).

Elles consistent en une augmentation du volume et de la durée de la distillation et en l'emploi de la technique colorimétrique de Michaels avec lecture à $420 \mathrm{~m} \mu$.

Ces modifications améliorent très notablement la reproductibilité du dosage de l'acide $\beta$-hy. droxybutyrique

\section{INTRODUCTION}

La détermination des corps cétoniques dans les liquides biologiques pose un certain nombre de problèmes de méthodologie. Plusieurs méthodes ont été proposées mais aucune ne donne entière satisfaction. Les tests qui peuvent rendre de grands services en clinique vétérinaire ou médicale ne sont pas quantitatifs. La méthode la plus satisfaisante pour l'esprit et la plus sélective est a priori, la méthode enzymatique WILliamson (I965). Mais la préparation et la purification de l'enzyme sont longues et délicates. On ne peut absolument éliminer la polyol-déshydrogénase et la malicodéshydrogénase si bien que, le fructose, le sorbitol et les malates perturbent le dosage.

Les méthodes gravimétriques, comme celle de VAN SLYKE (I9I7) utilisant 1e réactif de DENIGES ne sont précises que pour des concentrations élevées ; on n'observe pas de précipité pour des concentrations allant jusqu'à $5 \mathrm{mg} \mathrm{p}$. Ioo $\mathrm{ml}$. La sensibilité est améliorée par néphélométrie SHIPLEY et al., (I948). Mais les auteurs eux-mêmes 
estiment qu'avec des concentrations de l'ordre de I mg p. Ioo $\mathrm{ml}$, l'erreur peut atteindre I5 p. Ioo.

Les méthodes titrimétriques sont plus précises. La méthode de WEIChSEI,BAUM et al. (I94I) donne d'excellents résultats pour des concentrations proches de ro mg p. Ioo $\mathrm{ml}$; mais peu reproductibles pour des concentrations faibles.

Nous avons surtout étudié les méthodes colorimétriques qui se prêtent bien à des dosages en série et conviennent à la détermination de concentrations relativement faibles. Elles peuvent donc être utilisées pour des études sur animaux normaux.

\section{I. - DÉTERMINATION COLORIMÉTRIQUE DE L'ACÉTONE}

L'acétone donne avec l'aldśhyde salicylique en milieu alcalin, un produit de condensation de coloration rouge à orange. REID (I960) utilise cette réaction pour la détermination colorimétrique de l'acétone par lecture à $530 \mathrm{~m} \mu$. MrchaEIs et al. (I95 I) utilisent la réaction entre l'acétone et la 2-4 dinitrophénylhydrazine conduisant à la formation d'une hydrazone, extractible par le tétrachlorure de carbone et donnant un milieu coloré lisible à $420 \mathrm{~m} \mu$. Nous avons comparé ces deux méthodes à l'aide de solutions pures d'acétone de concentration connue. Les densités optiques observées sont reportées sur la figure $r$. Nous avons retenu la méthode de MrCHaELs qui suit mieux la loi de Beer que celle de RÉm.

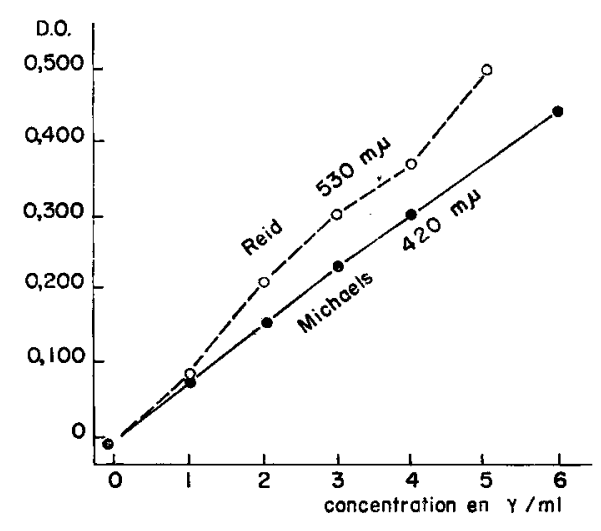

FIG. I.

\section{II. - TRANSFORMATION DES ACIDES ACÉTOACÉTIQUE ETT $\beta$-HYDROXYBU'TYRIQUE EN ACÉTONE}

I a transformation de l'acide acétoacétique en acétone ne pose pas de problèmes L'acide sulfurique l'hydrolyse en totalité et le rendement égale roo p. IOO. Il n'en est pas de même pour 1'acide $\beta$-hydroxybutyrique qui ne peut être dosé qu'après oxydation en acide acétoacétique qui est ensuite hydrolysé en acétone. Mais le rendement de ces réactions dépend des conditions d'oxydation et n'atteint jamais roo $p$. roo. 
Il faut donc opérer dans des conditions nettement définies. Aussi existe-t-il de nombreuses méthodes qui sont toutes basées sur le principe de l'oxydation par le bichromate de potassium.

MICHAELS et al. (I95I) réalisent l'oxydation en flacons bouchés hermétiquement et placés dans un bain-marie. La détermination colorimétrique se fait directement sans distillation et porte donc sur la totalité des corps cétoniques. Cette méthode ne permet pas l'évaluation séparée de l'acide $\beta$-hydroxybutyrique, mais uniquement par différence entre corps cétoniques totaux et somme acétoacétique + acétone mesurées sur des prises d'essai différentes ce qui multiplie les causes d'erreur.

THIN et ROBERTSON (I952) réalisent l'oxydation et l'hydrolyse à la température ambiante en cellules de Conway ; l'acétone diffuse dans l'enceinte intérieure et se combine à 1'aldéhyde salicylique. Cette méthode ne nous a pas donné satisfaction car nous n'avons pu empêcher l'altération de l'aldéhyde et obtenir un blanc stable.

GREENBERG et LESTER (I944) conduisent l'oxydation à chaud sous reflux et procèdent ensuite à une distillation également à chaud. Leur méthode a été améliorée par MAYES et RoBson (I957) qui réalisent reflux et distillation avec le même appareil. REID (I960), grâce à un appareil de distillation original, 1'a encore simplifiée en menant de pair reflux partiel et distillation. Nous avons étudié cette dernière méthode pour l'oxydation du $\beta$-hydroxybutyrate et la distillation de l'acétone formée que nous avons dosée par colorimétrie selon la méthode de MrchaELs.

L'hydrolyse de l'acide acétoacétique et sa distillation selon la méthode de REID (I960) sont très reproductibles et s'effectuent avec un rendement de Ioo p. Ioo. Nous n'avons par contre pas obtenu avec cette même méthode un rendement constant pour l'oxydation de l'acide $\beta$-hydroxybutyrique en solution pure ou en surcharge en milieu sang ou urine. Le tableau $\mathrm{I} a$ montre que le taux de récupération de la surcharge est très variable avec un volume de distillation de $5 \mathrm{ml}$ en 5 minutes préconisé par REID. Nous avons constaté qu'il restait encore de l'acide $\beta$-hydroxybutyrique non oxydé dans l'appareil après 5 minutes de distillation. Aussi avons-nous augmenté le temps

\section{TABLEAU I}

Comparaison des rendements de l'oxydation de la distillation et de la colorimétrie de l'acide hydroxybutyrique, par la technique originale de REID

(8 mesures)

\begin{tabular}{|c|c|c|c|c|c|c|}
\hline \multicolumn{4}{|c|}{ Tableau $1 a$} & \multicolumn{3}{|c|}{ Tableau $1 b$} \\
\hline $\begin{array}{l}\text { Volume de } \\
\text { distillation }\end{array}$ & \multicolumn{3}{|c|}{$5 \mathrm{ml} \times 5 \mathrm{mn}$} & \multicolumn{3}{|c|}{$10 \mathrm{ml} \times 10 \mathrm{mn}$} \\
\hline \multirow{2}{*}{ Rendement } & minimum & maximum & moyen & minimum & maximum & moyen \\
\hline & 38,5 & 78,0 & $62,2 \pm 15,6$ & 72,0 & 78,2 & $75,5 \pm 2,1$ \\
\hline
\end{tabular}

et le volume de celle-ci en les portant respectivement à Io $\mathrm{ml}$ et à Io minutes pour garder la même vitesse de distillation. Ceci nous a permis d'obtenir aussi bien en solution pure (tabl. I $b$ ) qu'en surcharge sur le sang ou l'urine, des taux de récupération satisfaisants (tabl. 2). 
Le rendement et la récupération des surcharges étant de $75 \mathrm{p}$. Ioo en moyenne pour l'acide $\beta$-hydroxybutyrique, il convient de multiplier par $4 / 3$ le résultat obtenu pour cet acide. Toutefois, les conditions de chauffage et la vitesse de distillation ont une influence sur ce rendement, il faut donc les standardiser et contrôler le rendement périodiquement au moyen de solutions pures.

\section{TABLEAU 2}

Récupération des surcharges d'acide hydroxybutyrique sur le sang (distillation $10 \mathrm{ml}$ ) par la technique de REID modifiée

\begin{tabular}{|c|c|c|c|c|}
\hline $\begin{array}{c}\text { Acétone p. } 100 \mathrm{ml} \\
\text { correspondant à la } \\
\text { D.O. lue } \\
(\mathrm{mg})\end{array}$ & $\begin{array}{c}\text { Surcharge (exprimée } \\
\text { en acétone) } \\
\text { (mg) }\end{array}$ & $\begin{array}{c}\text { Acétone p. } 100 \mathrm{ml} \\
\text { après surcharge } \\
(\mathrm{mg})\end{array}$ & $\begin{array}{l}\text { Surcharge récupérée } \\
\text { (mg) }\end{array}$ & $\begin{array}{c}\text { Taux } \\
\text { de récupération } \\
(\mathrm{mg})\end{array}$ \\
\hline 2,48 & 0,6 & 2,93 & 0,45 & 75,0 \\
\hline 2,78 & 0,6 & 3,24 & 0,46 & 76,7 \\
\hline 2,45 & 0,6 & 3,89 & 0,45 & 73,5 \\
\hline 2,03 & 1,8 & 3,36 & 1,33 & 73,8 \\
\hline 2,07 & 1,8 & 3,46 & 1,41 & 78,4 \\
\hline 2,14 & 1,8 & 3,44 & 1,30 & 72,2 \\
\hline 2,5 & 3,0 & 4,80 & 2,23 & 74,3 \\
\hline 2,44 & 3,0 & 4,62 & 2,18 & 72,7 \\
\hline 2,51 & 3,0 & 4,93 & 2,22 & 74,0 \\
\hline
\end{tabular}

\section{III. - MÉTHODOLOGIE ADOPTÉE}

\section{Réactifs}

$\mathrm{SO}_{4} \mathrm{Zn} 5$ p. $100(\mathrm{p} / \mathrm{v})$

$\mathrm{Ba}(\mathrm{OH})_{2}$ environ $0,3 \mathrm{~N}$

$\mathrm{SO}_{4} \mathrm{Cu} 10 \mathrm{p}$. $100\left(\mathrm{SO}_{4} \mathrm{Cu}_{5} \mathrm{H}_{2} \mathrm{O} \mathrm{p} / \mathrm{v}\right)$

Lait de chaux (roo $\mathrm{g} \mathrm{CaO}$ en suspension par litre)

$\mathrm{SO}_{4} \mathrm{H}_{8} 7 \mathrm{~N}$

$\mathrm{K}_{2} \mathrm{Cr}_{2} \mathrm{O}_{7}$ 0,2 p. $100(\mathrm{p} / \mathrm{v})$

2-4 Dinitrophenylhydrazine 0,1 p. 100 dans $\mathrm{HCl} 2 \mathrm{~N}$

$\mathrm{NaOH}$ I0,5 N

$\mathrm{CCl}_{4}$

\section{Défécation}

A $5 \mathrm{ml}$ de sang ajouter $10 \mathrm{ml}$ de solution de $\mathrm{ZnSO}_{4}$ puis la quantité équivalente de $\mathrm{Ba}(\mathrm{OH})_{2}$ et de l'eau distillée pour obtenir une dilution de $\mathrm{I} / \mathrm{I} 0$ à $\mathrm{I} / 80$. Laisser reposer au moins 30 minutes et filtrer ou centrifuger SomOGYI, (1945). Si le liquide à déféquer contient plus de $2 \mathrm{~m} / 1$ de sucres réducteurs, il est préférable d'employer la solution de $\mathrm{CuSO}_{4}$ et le lait de chaux LEPPER, (I938).

\section{Distillation}

Porter à ébullition $8 \mathrm{ml}$ de $\mathrm{SO}_{4} \mathrm{H}_{2} 7 \mathrm{~N}$ (pour avoir une ébullition régulière ; employer un petit tube de verre fermé à une extrémité et à $0,5 \mathrm{~cm}$ de l'autre extrémité pour emprisonner une petite bulle d'air). Introduire la prise d'essai (Io à $50 \gamma$ de corps cétoniques totaux exprimés en acétone) après avoir vu perler quelques gouttes de distillat, au moyen du tube capillaire, sans interrompre l'ébullition.

Régler le dispositif de chauffage de façon à distiller à vitesse constante 10 ml en 10 minutes. Cette première fraction correspond à l'acétone et à l'acide acétoacétique. 
Rajouter par le capillaire $5 \mathrm{ml} \mathrm{de} \mathrm{K}_{2} \mathrm{Cr}_{2} \mathrm{O}_{7}$ et distiller à nouveau Io $\mathrm{ml}$ en Io $\mathrm{mn}$. Cette deuxième fraction correspond à l'acide $\beta$-hydroxybutyrique.

\section{Colorimétrie}

Ajouter $5 \mathrm{ml}$ de dinitrophénylhydrazine puis $5 \mathrm{ml}$ exactement mesurés de $\mathrm{CCl}_{4}$. Agiter Io minutes Additionner $3 \mathrm{ml}$ de soude et agiter 5 minutes.

Lire la densité optique à $420 \mathrm{~m} \mu$ par rapport à un blanc (distillat remplacé par eau distillée) et à un standard (distillat remplacé par une solution connue d'acétone).

Reçu pour publication en novembre I967.

\section{SUMMARY}

\section{THE DETERMINATION OF KETONE BODIES IN BLOOD, URINE AND RUMINAL CONTENTS}

A modified Reid's method was applied to the estimation of ketone bodies in blood, urine and ruminal contents.

The modifications consist in an increase in the volume and duration of the distillation and the use of Michaels's colorimetric technique with readings taken at $420 \mathrm{~m} \mu$.

These modifications consistently improved the reproducibility of estimation of the $\beta$-hydroxybutyric acid.

\section{RÉFÉRENCES BIBLIOGRAPHIQUES}

GreEnBerg L. A., Lester D. I944. A micromethod for the determination of acetone and ketone bodies. J. Biol. Chem., 154, i78-190.

LEPPER W. 1938. Technique de dosage des acides acétiques, butyrique et lactique dans les ensilages. Tierernähr. U. Juttermitl., 1, 187-196.

Mayes P. A., RoBson W. I957. The determination of ketone bodies. Biochem. J., 67, II-I4.

McCandless, Dye., I950. cité dans Nutrition animale Jacquot R., Leroy A. M., Simonet H., Courvoisier F., Weber M., Lebars H. Volume II. Données générales sur la nutrition et l'alimentation. Tome r. Métabolismes et transits par JACQUOT R., LE BARS H., SIMONET H., p. 625.

Michaels G. D., Margen S., Liebert G., Kinsell L. W., 195I. Studies in fat metabolism; the colorimetric determination of ketone bodies in biological fluids. J. Clin. Invest., 30, 1483-1485.

REID R. L. 1960. The determination of ketone bodies in blood. The analyst, 85, 265-272.

ShIPLEY R. A., Long C. N. H., I938. The relation of ketonemia to ketonuria in the rat. Biochem. $J$., 32, $2242-2246$.

SoMOGYI M., I945. A new reagent for the determination of sugars. J. Biol. Chem., 160, 6I-68.

THIN C., RoBERTSON A. 1952. The estimation of acetone bodies. Biochem. J. 51, $218-223$.

VAN SLYKE D. D., I9I7. Studies of acidosis. The determination of $\beta$-hydroxybutyrique acid, aceto and acetone in urine. J. Biol. Chem., $32,455^{-462 .}$

WeichSELbaum T. E., Somogyi M. I94I. A new method for determination of small amounts of ketone bodies. J. Biol. Chem., 140, 5-10.

Willimson D. K. H., Mellamby J., KREBS A., 1965. Enzymatic determination of D (-) $\beta$-hydroxybutiric acid and acetoacetic in blood. $96,210-217$. 